\title{
Estimation of the Unknown Parameters for the Compound Rayleigh Distribution Based on Progressive First-Failure-Censored Sampling
}

\author{
Tahani A. Abushal \\ Department of Mathematics, Umm Al-Qura University, Makkah Al-Mukarramah, KSA \\ E-mail: tabushal@yahoo.com \\ Received August 2, 2011; revised September 5, 2011; accepted September 17, 2011
}

\begin{abstract}
This article considers estimation of the unknown parameters for the compound Rayleigh distribution (CRD) based on a new life test plan called a progressive first failure-censored plan introduced by $\mathrm{Wu}$ and Kus (2009). We consider the maximum likelihood and Bayesian inference of the unknown parameters of the model, as well as the reliability and hazard rate functions. This was done using the conjugate prior for the shape parameter, and discrete prior for the scale parameter. The Bayes estimators have been obtained relative to both symmetric (squared error) and asymmetric (LINEX and general entropy (GE) loss functions. It has been seen that the symmetric and asymmetric Bayes estimators are obtained in closed forms. Also, based on this new censoring scheme, approximate confidence intervals for the parameters of CRD are developed. A practical example using real data set was used for illustration. Finally, to assess the performance of the proposed estimators, some numerical results using Monte Carlo simulation study were reported.
\end{abstract}

Keywords: Compound Rayleigh Distribution, Progressive First-Failure Censored Scheme, Bayesian and Non-Bayesian Estimations, Approximate Confidence Intervals

\section{Introduction}

There are many scenarios in life-testing and reliability experiments whose units are lost or removed from experimentation before failure. The loss may occur unintentionally, or it may have been designed so in the study. However, in many situations, the removal of units prior to failure is preplanned in order to provide saving in terms of time and cost associated with testing. There are many types of censored test, the most common censoring schemes are type-I and type-II censoring, but using this types of censoring can not allow for units to be removed from the test at any other point than the final termination point. However, if an experimenter desires to remove surviving units at points other than the final termination point of the life test, these two traditional censoring schemes will not be of use to the experimenter. The allowance of removing surviving units from the test before the final termination point is desirable, as in the case of studies of wear, in which the study of the actual aging process requires units to be fully disassembled at different stages of the experiment. In addition, when a com- promise between the reduced time of experimentation and the observation of at least some extreme lifetimes is sought, such an allowance is also desirable. These reasons lead us into the area of progressive censoring.

It is well known that one of the primary goals of progressive censoring is to save some live units for other tests, which is particularly useful when the units being tested are very expensive. [1] mentioned that the inference is feasible, and practical when the sample data are gathered according to a Type-II progressively censored study experimental scheme. Statistical inferences on the parameters of failure time distributions under progressive censoring have been studied by several authors such as [1-12]. A recent account on progressive censoring schemes can be found in the book by [13], or in the excellent review by [14].

[15] described a life test in which the experimenter might decide to group the test units into several sets, each as an assembly of test units, and then run all the test units simultaneously until occurrence the first failure in each group. Such a censoring scheme is called first-failure censoring. [16] discussed a sampling plan for a bear- 
ing manufacturer. The bearing test engineer decided to save test time by testing 50 bearings in sets of 10 each, and the first-failure times from each group were observed. $[17,18]$ discussed some inferences based on firstfailure-censored sampling from both the Gompertz distribution and Burr-XII distribution, respectively. Also see $[19,20]$. Note that a first-failure-censoring scheme is terminated when the first failure in each set is observed. If an experimenter desires to remove some sets of test units before observing the first failures in these sets this life test plan is called a progressive first-failure-censoring scheme which recently introduced by [21].

The two-parameter compound Rayleigh distribution (which is denoted by $\operatorname{CRD}(\alpha, \beta)$ ) provides a population model which is useful in several areas of statistics, including life testing and reliability. The probability density function $(p d f)$, and the cumulative distribution function (cdf) of CRD $(\alpha, \beta)$ are given, respectively, by

$$
\begin{gathered}
f(x)=2 \alpha \beta^{\alpha} x\left(\beta+x^{2}\right)^{-(\alpha+1)}, \\
x>0, \beta>0, \alpha>0, \\
F(x)=1-\beta^{\alpha}\left(\beta+x^{2}\right)^{-\alpha},
\end{gathered}
$$

and the reliability and failure rate functions, at some $t$, are

$$
\begin{gathered}
S(t)=\beta^{\alpha}\left(\beta+t^{2}\right)^{-\alpha}, t>0, \\
H(t)=\frac{2 \alpha t}{\beta+t^{2}}, t>0,
\end{gathered}
$$

where $\alpha$ and $\beta$ are the shape and the scale parameter respectively.

The compound Rayleigh distribution (CRD) is a special case of the 3- parameter Burr type XII distribution, which has a PDF of the form. The 2- parameter version of this distribution (with) was studied by several authors, such as [22-26], among others.

The main aim of this article is to focus on the designing problem of a progressive first-failure censoring life test with a compound Rayleigh failure time distribution. The rest of this article is organized as follows. In Section 2 , the formulation of a progressive first-failure-censoring scheme is described. The ML estimations with the approximate confidence intervals of the parameters are obtained in Section 3. Bayesian estimations of the parameters, reliability and hazard rate functions of CRD based on progressive first-failure-censoring scheme are investigated in Section 4. In Section 5, for illustrative purposes, we performed a real data analysis. A simulation study in order to give an assessment of the performance of the estimation methods are presented in Section 6 . Finally we conclude the paper in Section 7.

\section{A Progressive First-Failure-Censoring Scheme}

In this section, first-failure-censoring scheme is combined with the progressive censoring scheme as in [21]. Suppose that $n$ independent groups with $k$ items within each group are put in a life test, $R_{1}$ groups and the group in which the first failure is observed are randomly removed from the test as soon as the first failure (say $X_{1: m: n: k}^{\mathrm{R}}$ ) has occurred, $R_{2}$ groups and the group in which the second first failure is observed are randomly removed from the test when the second failure (say $\left.X_{2: m: n: k}^{\mathrm{R}}\right)$ has occurred, and finally $R_{m}(m \leq n)$ groups and the group in which the $m$-th first failure is observed are randomly removed from the test as soon as the $m$-th failure (say $X_{m: m: n: k}^{\mathbf{R}}$ ) has occurred. The $X_{1: m: n: k}^{\mathbf{R}}$ $<X_{2: m: n: k}^{\mathbf{R}}<\cdots<X_{m: m: n: k}^{\mathbf{R}}$ are called progressively firstfailure-censored order statistics with the progressive censoring scheme $\mathbf{R}=\left(R_{1}, R_{2}, \cdots, R_{m}\right)$. It is clear that $n=m+\sum_{j=1}^{m} R_{i}$. If the failure times of the $n \times k$ items originally in the test are from a continuous population with distribution function $F(x)$ and probability density function $f(x)$, the joint probability density function for $X_{1: m: n: k}^{\mathbf{R}}, X_{2: m: n: k}^{\mathbf{R}}, \cdots, X_{m: m: n: k}^{\mathbf{R}}$ is given by

$$
\begin{aligned}
& f_{1,2, \ldots, m}\left(x_{1: m: n: k}^{\mathbf{R}}, x_{2: m: n: k}^{\mathbf{R}}, \ldots, X_{m: m: n: k}^{\mathbf{R}}\right) \\
= & C k \prod_{j=1}^{m} f\left(x_{j: m: n: k}^{\mathbf{R}}\right)\left[1-F\left(x_{j: m: n: k}^{\mathbf{R}}\right)\right]^{k\left(\mathbf{R}_{j}+1\right)-1}, \\
0< & x_{1: m: n: k}^{\mathbf{R}}<x_{2: m: n: k}^{\mathbf{R}}<\ldots<x_{m: m: n: k}^{\mathbf{R}}<\infty,
\end{aligned}
$$

where

$$
\begin{aligned}
C= & n\left(n-R_{1}-1\right)\left(n-R_{1}-R_{2}-1\right) \cdots \\
& \left(n-R_{1}-R_{2}-\cdots R_{m-1}-m+1\right) .
\end{aligned}
$$

It should be noted that $X_{1 ; m, n, k}^{\mathbf{R}}, X_{2 ; m, n, k}^{\mathbf{R}}, \cdots, X_{m ; m, n, k}^{\mathbf{R}}$ can be viewed as a progressive type II censored sample from a population with distribution function 1-(1$F(x))^{k}$. For this reason, results for progressive type II censored order statistics can be extended to progressive first-failure censored order statistics easily. Also, the progressive first-failure censored plan has advantages in terms of reducing the test time, in which more items are used, but only $m$ of $n \times k$ items are failures.

\section{Maximum Likelihood Estimation}

Based on progressively first-failure-censored sample $X_{i: m: n: k}^{\mathbf{R}}, \quad i=1,2, \cdots, m$, with censoring scheme $R$ is drawn from the CRD (1). For convenience, we will denote the observed values of such sample by $X_{i}, i=1$, $2, \cdots, m$. From (5), the likelihood function is given by 


$$
\begin{aligned}
L(\beta, \alpha) & =C(2 k \alpha)^{m} \beta^{\alpha k n} \\
& \exp \left(-\alpha k \sum_{i=1}^{m}\left(R_{i}+1\right) \log \left[\beta+x_{i}^{2}\right]\right) \prod_{i=1}^{m} \frac{x_{i}}{\beta+x_{i}^{2}},
\end{aligned}
$$

where $C$ is defined in (6) The log-likelihood function is given by

$$
\begin{aligned}
\ell(\beta, \alpha) & =\log \left[C(2 k)^{m}\right]+m \log \alpha+\alpha k n \log \beta \\
& +\sum_{i=1}^{m} \log \left[\frac{x_{i}}{\beta+x_{i}^{2}}\right]-\alpha k \sum_{i=1}^{m}\left(R_{i}+1\right) \log \left[\beta+x_{i}^{2}\right] .
\end{aligned}
$$

Calculating the first partial derivative of (8) with respect to $\alpha$ and $\beta$ and equating to zero, we obtain the likelihood equations

$$
\frac{\partial \ell(\beta, \alpha)}{\partial \alpha}=\frac{m}{\alpha}+k n \log \beta-k \sum_{i=1}^{m}\left(R_{i}+1\right) \log \left(\beta+x_{i}^{2}\right)=0,
$$

and

$$
\frac{\partial \ell(\beta, \alpha)}{\partial \beta}=\frac{\alpha k n}{\beta}-\sum_{i=1}^{m} \frac{1}{\beta+x_{i}^{2}}+\alpha k \sum_{i=1}^{m} \frac{\left(R_{i}+1\right)}{\left(\beta+x_{i}^{2}\right)}=0
$$

From (9), we have

$$
\hat{\alpha}(\beta)=\frac{m}{k\left[\sum_{i=1}^{m}\left(R_{i}+1\right) \log \left(\beta+x_{i}^{2}\right)-n \log \beta\right]}
$$

From (10) and (11), we have

$$
\frac{\frac{n}{\hat{\beta}}-\sum_{i=1}^{m} \frac{\left(R_{i}+1\right)}{\hat{\beta}+x_{i}^{2}}}{\sum_{i=1}^{m}\left(R_{i}+1\right) \log \left(\hat{\beta}+x_{i}^{2}\right)-n \log \hat{\beta}}-\frac{1}{m} \sum_{i=1}^{m} \frac{1}{\hat{\beta}+x_{i}^{2}}=0
$$

Newton-Raphson iteration is employed to solve (12). The corresponding MLE's of the reliability function $S(t)$, and hazard rate function $H(t)$, are given respectively by (3) and (4) after replacing $\beta$ and $\alpha$ by their MLE's $\hat{\beta}$ and $\hat{\alpha}$. To obtain a starting value for the root finding method, we can use the graphical method discussed in [27].

\section{Approximate Interval Estimation}

From the log-likelihood function in (8), we have

$$
\begin{gathered}
\frac{\partial^{2} \ell(\beta, \alpha)}{\partial \beta^{2}}=\frac{-\alpha k n}{\beta^{2}}+\sum_{i=1}^{m} \frac{1}{\left(\beta+x_{i}^{2}\right)^{2}}-\alpha k \sum_{i=1}^{m} \frac{\left(R_{i}+1\right)}{\left(\beta+x_{i}^{2}\right)^{2}} \\
\frac{\partial^{2} \ell(\beta, \alpha)}{\partial \alpha^{2}}=\frac{-m}{\alpha^{2}}
\end{gathered}
$$

and

$$
\frac{\partial^{2} \ell(\beta, \alpha)}{\partial \beta \partial \alpha}=\frac{\partial^{2} \ell(\beta, \alpha)}{\partial \alpha \partial \beta}=\frac{k n}{\beta}+k \sum_{i=1}^{m} \frac{\left(R_{i}+1\right)}{\left(\beta+x_{i}^{2}\right)} .
$$

Let $\quad X_{1: m: n: k}^{\mathbf{R}}<X_{2: m: n: k}^{\mathbf{R}}<\cdots<X_{m: m: n: k}^{\mathbf{R}} \quad$ denote a progressively first-failure-censored sample from the CRD with parameters $\alpha$ and $\beta$. The Fisher information matrix $I(\beta, \alpha)$ is then obtained by taking expectation of minus Equations (13)-(15). Under some mild regularity conditions, $(\hat{\beta}, \hat{\alpha})$ is approximately bivariate normal with mean $(\beta, \alpha)$ and covariance matrix $I^{-1}(\beta, \alpha)$. In practice, we usually estimate $I^{-1}(\beta, \alpha)$ by $I^{-1}(\hat{\beta}, \hat{\alpha})$. A simpler and equally valied procedure is to use the approximation

$$
(\hat{\beta}, \hat{\alpha}) \sim N\left((\beta, \alpha), I_{0}^{-1}(\hat{\beta}, \hat{\alpha})\right),
$$

where $I_{0}(\beta, \alpha)$ is the observed information matrix given by

$$
I_{0}(\hat{\lambda}, \hat{\beta})=\left[\begin{array}{cc}
-\frac{\partial^{2} \ell(\beta, \alpha)}{\partial \beta^{2}} & -\frac{\partial^{2} \ell(\beta, \alpha)}{\partial \beta \partial \alpha} \\
-\frac{\partial^{2} \ell(\beta, \alpha)}{\partial \alpha \partial \beta} & -\frac{\partial^{2} \ell(\beta, \alpha)}{\partial \alpha^{2}}
\end{array}\right]_{(\hat{\beta}, \hat{\alpha})} .
$$

Approximate confidence intervals for $\beta$ and $\alpha$ can be found by to be bivariate normal with mean $(\beta, \alpha)$ and covariance matrix $I_{0}^{-1}(\hat{\beta}, \hat{\alpha})$. Thus, a $100(1-\gamma) \%$ approximate confidence intervals for $\beta$ and $\alpha$ are

$$
\hat{\beta} \mp z_{\frac{\gamma}{2}} \sqrt{v_{11}} \text { and } \hat{\alpha} \mp z_{\frac{\gamma}{2}} \sqrt{v_{22}}
$$

respectively, where $v_{11}$ and $v_{22}$ are the elements on the main diagonal of the covariance matrix $I_{0}^{-1}(\hat{\beta}, \hat{\alpha})$ and $z_{\frac{\gamma}{2}}$ is the percentile of the standard normal distribution with right-tail probability $\frac{\gamma}{2}$.

\section{Bayes Estimation}

In this section, we present the posterior densities of the parameters $\beta$ and $\alpha$, and obtain symmetric and asymmetric Bayes estimators for the parameters, reliability and hazard rate functions.

\subsection{The Loss Function}

For Bayesian approach, in order to select a single value as representing our "best" estimators of the unknown parameter, a loss function must be specified. A wide variety of loss functions has been developed in literature to describe various types of loss structures. The symmetric square-error loss (SE) is one of the most popular loss functions. It is widely employed in the inference, but its application is motivated by its good mathematical prop- 
erties, not by its applicability to representing a true loss structure. A loss function should represent the consequences of different errors. There are situations where over- and under-estimation can lead to different consequences. For example, when we estimate the average reliable working life of the components of a spaceship or an aircraft, over-estimation is usually more serious than underestimation. Being symmetric, the SE loss equally penalizes over- and under-estimation of the same magnitude. A useful asymmetric loss known as the LINEX loss function, was introduced by [28], and was widely used in several papers, see for example, [5,6,10,11,2830]. This function rises approximately exponentially on one side of zero, and approximately linearly on the other side. Under the assumption that the minimal loss occurs at $\tilde{u}=u$, the LINEX loss function for $u=u(\beta, \alpha)$ can be expressed as

$$
L_{1}(\Delta) \propto \mathrm{e}^{a \Delta}-a \Delta-1, \quad a \neq 0,
$$

where $\Delta=(\tilde{u}-u), \tilde{u}$ is an estimate of $u$.

The sign, and magnitude of the shape parameter $a$ represent the direction and degree of symmetry respectively (if $a>0$, overestimation is more serious than underestimation and $a<0$ means the opposite). For $a$ close to zero, the LINEX loss function is approximately the squared error loss (SEL) and therefore almost symmetric.

The posterior expectation of the LINEX loss function of (19) is

$$
\begin{aligned}
E_{u}(L(\tilde{u}-u)) & \propto \exp (a \tilde{u}) \cdot E_{u}[\exp (a u)] \\
& -a \cdot\left(\tilde{u}-E_{u}[u]\right)-1 .
\end{aligned}
$$

where $E_{u}$ is denotes posterior expectation with respect to the posterior $p d f(u)$. The Bayes estimator $\tilde{u}_{B L}$ of $u$ under the LINEX loss function is the value $\tilde{u}$, which minimizes (20), it is

$$
\tilde{u}_{B L}=-\frac{1}{a} \log \left(E_{u}[\exp (-a u)]\right)
$$

provided that the expectation $E_{u}[\exp (-a u)]$ exists and is finite.

Another useful asymmetric loss function is the general entropy loss (GEL)

$$
L_{2}(\hat{u}, u) \propto\left(\frac{\tilde{u}}{u}\right)^{q}-q \log \left(\frac{\tilde{u}}{u}\right)-1
$$

whose minimum occurs at $\tilde{u}=u$. This loss function is a generalization of the entropy-loss used in several papers where $q=1$ by [31] and [32]. When $q>0$, a positive error $(\tilde{u}>u)$ causes more serious consequences than a negative error. The Bayes estimate $\tilde{u}_{B G}$ of $u$ under
GE loss (22) is

$$
\tilde{u}_{B G}=\left(E_{u}\left[u^{-q}\right]\right)^{\frac{-1}{q}}
$$

provided that $E_{u}\left[u^{-q}\right]$ exists, and is finite.

\subsection{Prior Distribution and Posterior Analysis}

In this section we first describe the prior information needed for the Bayesian analysis of the unknown parameters. When the parameters $\beta$ and $\alpha$ are assumed to be unknown, the following idea of [33], we assume that the parameter $\beta$ has a discrete prior, while the conditional distribution of $\alpha$ given $\beta=\beta_{j}$ has a conjugate gamma prior. Now, we suppose that the parameter $\beta$ is restricted to a finite number of values, say $\beta_{1}, \beta_{2}, \cdots, \beta_{N}$ in the interval $(0, \infty)$, i.e.

$p\left(\beta=\beta_{j}\right)=e_{j}, \sum_{j=1}^{N} e_{j}=1$ and $0 \leq e_{j} \leq 1$,

$(j=1,2, \cdots, N)$. Further, suppose that conditional upon $\beta=\beta_{j}, \quad j=1,2, \quad \cdots, N, \alpha$ has a natural conjugate gamma $\left(a_{j}, b_{j}\right)$ prior, with density

$$
\begin{gathered}
\pi_{1}\left(\alpha \mid \beta=\beta_{j}\right)=\frac{b_{j}^{a_{j}} \alpha^{a_{j}-1} \exp \left(-b_{j} \alpha\right)}{\Gamma\left(a_{j}\right)}, \\
\alpha>0, a_{j}, b_{j}>0 .
\end{gathered}
$$

Combining the likelihood function in (5), and prior density (24), we obtain the marginal posterior probability of $\alpha$ conditional $\beta=\beta_{j}$

$$
\pi_{1}^{*}\left(\alpha \mid \beta=\beta_{j}\right)=\frac{T_{j}^{\omega_{j}} \alpha^{\omega_{j}-1} \exp \left(-\alpha T_{j}\right)}{\Gamma\left(\omega_{j}\right)},
$$

where

$$
T_{j}=b_{j}+k \sum_{i=1}^{m}\left(R_{i}+1\right)\left[\log \left(\beta+x_{i}^{2}\right)-\log \beta\right], \quad \omega_{j}=m+a_{j} .
$$

On applying the discrete version of Bayes theorem, the marginal posterior probability distribution of $\beta$ is given by

$$
\begin{aligned}
p_{j} & =\operatorname{Pr}\left(\beta=\beta_{j}\right) \\
& =\rho \int_{\alpha} e_{j} L\left(\underline{x} ; \beta_{j}, \alpha\right) \pi_{1}\left(\alpha \mid \beta=\beta_{j}\right) \mathrm{d} \alpha \\
& =\rho \frac{e_{j} b_{j}^{a_{j}} \Gamma\left(\omega_{j}\right) \prod_{i=1}^{m} \frac{x_{i}}{\beta_{j}+x_{i}^{2}}}{T_{j}^{\omega_{j}} \Gamma\left(a_{j}\right)},
\end{aligned}
$$

where $\omega_{j}$ and $T_{j}$ given by (26) and $\rho$ is a normalized constant given by 


$$
e^{-1}=\sum_{j=1}^{N} \frac{e_{j} b_{j}^{a_{j}} \Gamma\left(\omega_{j}\right) \prod_{i=1}^{m} \frac{x_{i}}{\beta_{j}+x_{i}^{2}}}{T_{j}^{\omega_{j}} \Gamma\left(a_{j}\right)} .
$$

The joint posterior probability of $\alpha$ and $\beta$ is

$$
\pi_{1}^{*}(\alpha, \beta)=P_{j} \frac{T_{j}^{\omega_{j}} \alpha^{\omega_{j}-1} \exp \left(-\alpha T_{j}\right)}{\Gamma\left(\omega_{j}\right)}, \alpha>0, a_{j}, b_{j}>0 .
$$

\subsection{Symmetric Bayes Estimation}

By using posterior denisties in (25) and (27), the Bayes estimators $\tilde{\beta}_{B S}, \tilde{\alpha}_{B S}, \tilde{S}_{B S}$, and $\tilde{H}_{B S}$ of the parameters $\beta, \alpha$, reliability function $S(t)$, and hazard rate function $H(t)$ relative to ESL function, are, respectively

$$
\begin{gathered}
\tilde{\beta}_{B S}=\sum_{j=1}^{N} \beta_{j} P_{j}, \quad \tilde{\alpha}_{B S}=\sum_{j=1}^{N} P_{j} \frac{\omega_{j}}{T_{j}}, \\
\tilde{S}_{B S}(t)=\sum_{j=1}^{N} P_{j}\left[1+\frac{\log \left(1+\frac{t^{2}}{\beta_{j}}\right)}{T_{j}}\right]^{-\omega_{j}},
\end{gathered}
$$

and

$$
\tilde{H}_{B S}(t)=2 t \sum_{j=1}^{N} \frac{P_{j} \omega_{j}}{T_{j}\left(\beta+t^{2}\right)}
$$

\subsection{Asymmetric Bayes Estimation}

\subsubsection{LINEX Loss Function}

Based on progressively first-failure censored data, the Bayes estimators $\tilde{\beta}_{B L}, \tilde{\alpha}_{B L}, \tilde{S}_{B L}$, and $\tilde{H}_{B L}$ of the parameters $\beta, \alpha, \quad$ reliability function $S(t)$, and hazard rate function $H(t)$ relative to LINEX loss function, are, respectively

$$
\begin{gathered}
\tilde{\beta}_{B L}=-\frac{1}{\theta} \log \left[\sum_{j=1}^{N} P_{j} \exp \left(-\theta \beta_{j}\right)\right], \\
\tilde{\alpha}_{B L}=-\frac{1}{\theta} \log \left[\sum_{j=1}^{N} P_{j}\left(1+\frac{\theta}{T_{j}}\right)^{-\omega_{j}}\right], \\
\tilde{S}_{B L}=-\frac{1}{\theta} \log \left[\sum_{j=1}^{N} \sum_{s=0}^{\infty} \frac{(-\theta)^{s}}{s !} P_{j}\left(1+\frac{s \log \left(1+\frac{t^{2}}{\beta_{j}}\right)}{T_{j}}\right)^{-\omega_{j}}\right]
\end{gathered}
$$

and

$$
\tilde{H}_{B L}=-\frac{1}{\theta} \log \left[\sum_{j=1}^{N} P_{j}\left(1+\frac{2 \theta t}{T_{j}\left(\beta_{j}+t^{2}\right)}\right)^{-\omega_{j}}\right] .
$$

\subsubsection{GE Loss Function}

Also, by using posterior denisties (25) and (27), the Bayes estimators $\tilde{\beta}_{B G}, \tilde{\alpha}_{B G}, \tilde{S}_{B G}$, and $\tilde{H}_{B G}$ of the parameters $\beta, \alpha, S(t)$, and $H(t)$, relative to GE loss function, are, respectively

$$
\begin{gathered}
\tilde{\beta}_{B G}=\left[\sum_{j=1}^{N} \beta_{j}^{-q} P_{j}\right]^{(-1 / q)}, \\
\tilde{\alpha}_{B G}=\left[\sum_{j=1}^{N} P_{j} \frac{\left(T_{j}\right) \Gamma\left(\omega_{j}-q\right)}{\Gamma\left(\omega_{j}\right)}\right]^{(-1 / q)}, \\
\tilde{S}_{B G}=\left[\sum_{j=1}^{N} P_{j}\left(1-\frac{q \log \left(1+\frac{t^{2}}{\beta_{j}}\right)}{T_{j}}\right]^{-\omega_{j}}\right]^{(-1 / q)},
\end{gathered}
$$

and

$$
\tilde{H}_{B G}=\left[\sum_{j=1}^{N} P_{j}\left(\frac{2 t}{\beta_{j}+t^{2}}\right)^{-q} \times \frac{\left(T_{j}\right) \Gamma\left(\omega_{j}-q\right)}{\Gamma\left(\omega_{j}\right)}\right]^{(-1 / q)} .
$$

To implement the calculations in this subsections, it is first necessary to elicit the values of $\left(\beta_{j}, e_{j}\right)$ and the hyperparameters $\left(a_{j}, b_{j}\right)$ in the prior (25), for $j=1,2$, $\cdots, N$. The former paris of values are fairly straightforward to specify, but for $\left(a_{j}, b_{j}\right)$ it is necessary to condition prior beliefs about $\alpha$ on each $\beta_{j}$ in turn, and this can be difficult in practice, an alternative method for obtaining the values $\left(a_{j}, b_{j}\right)$ can be based on the expected value of the reliability function $S(t)$ conditional on $\beta=\beta_{j}$, see [34], which is given using (25) by

$$
\begin{aligned}
E_{\alpha \mid \beta}\left(S(t) \mid \beta_{j}\right) & =\left[1+\frac{\log \left(\beta_{j}+t^{2}\right)-\log \beta_{j}}{b_{j}}\right]^{-a_{j}}, \\
\alpha & >0, a_{j}, b_{j}>0 .
\end{aligned}
$$

Now, suppose that prior beliefs about the lifetime distribution enable one to specify two values $\left(S\left(t_{1}\right), t_{1}\right)$, $\left(S\left(t_{2}\right), t_{2}\right)$. Thus, for these two prior values $S\left(t=t_{1}\right)$ and $S\left(t=t_{2}\right)$, the values of $a_{j}$ and $b_{j}$ for each value $\beta_{j}$ can be obtained numerically from (43). If no prior beliefs, a nonparametric procedure can be used to estimate the corresponding two different values of $S(t)$, 
see [35].

\section{Data Analysis}

For illustrative purposes, we performed a real data analysis. The original data is a subset of data reported by $[6,36]$, represent the survival times in years of a group of patients given chemotherapy treatment alone. The data consisting of 46 survival times (in years) for 46 patients are: $0.047,0.115,0.121,0.132,0.164,0.197,0.203$, $0.260,0.282,0.296,0.334,0.395,0.458,0.466,0.501$, $0.507,0.529,0.534,0.540,0.570,0.641,0.644,0.696$, $0.841,0.863,1.099,1.219,1.271,1.326,1.447,1.485$, $1.553,1.581,1.589,2.178,2.343,2.416,2.444,2.825$, $2.830,3.578,3.658,3.743,3.978,4.003,4.033$. [6,36] show that the Compound Rayleigh model is acceptable for these data. Now, we suppose that the forty six patients are randomly grouped into 23 sets, with two patients in each $(k=2)$, and the survival times for all sets are observed and listed in ascending order in Table 1. In this example, based on survival times of the 23 sets given in Table 1, with $(m=15 ; n=23 ; k=2)$ and the censoring scheme $R=\{2,0,0,2,0,1,0,0,2,0,0,0,0,0,1\}$, we obtain the following progressive first-fauilure censoring data: $0.047,0.115,0.121,0.164,0.197,0.26$, $0.282,0.334,0.395,0.458,0.529,0.534,0.641,0.696$,
1.099 .

To compute the Bayes estimates. Firstly, we estimate two values of the reliability function using a nonparametric procedures. $S\left(t_{i}=X_{i ; m, n, k}^{R}\right)=\frac{(m-i+0.625)}{m+0.25}, i$ $=1,2, \cdots, m$, see [37]. In our case, we use $S\left(t_{1}=0.121\right)$ $=0.828$ and $S\left(t_{2}=0.197\right)=0.697$. These two prior probabilities are substituted into (39), where $a_{j}$ and $b_{j}$ are solved numerically for each given $\beta_{j}, j=1,2, \cdots$, 10 using Newton-Raphson method. Table 2 summarized the values of $a_{j}, b_{j}$ and $P_{j}$ for each given $\beta_{j}$ and $e_{j}, j=1,2, \cdots, 10$.

Using our results in Sections 3 and 4, the MLE estimates and the Bayes estimates of $\beta, \alpha, S(t)$ and $H(t)$ have been computed and the results are displayed in Table 3. By using (18) the $95 \%$ approximate confidence interval of $\beta$ and $\alpha$ are, respectively $(-0.3148$, $0.8027)$ and $(-0.3958,1.7547)$.

\section{Simulation Study}

Since the performance of the different methods cannot be compared theoretically, we perform Monte Carlo simula-

Table 1. Randomly grouped sets using data from Stablein (1981).

\begin{tabular}{|c|c|c|c|c|c|c|c|c|c|c|c|}
\hline 1 & 2 & 3 & 4 & 5 & 6 & 7 & 8 & 9 & 10 & 11 & 12 \\
\hline 0.197 & 0.534 & 0.115 & 0.296 & 0.121 & 0.466 & 0.529 & 1.447 & 0.863 & 0.132 & 0.395 & 0.696 \\
\hline 2.825 & 3.658 & 3.978 & 3.743 & 2.343 & 2.178 & 0.54 & 4.003 & 1.553 & 1.485 & 2.83 & 2.416 \\
\hline 13 & 14 & 15 & 16 & 17 & 18 & 19 & 20 & 21 & 22 & 23 & \\
\hline 0.26 & 1.099 & 0.501 & 0.458 & 0.641 & 0.334 & 0.57 & 0.164 & 0.203 & 0.282 & 0.047 & \\
\hline 1.271 & 1.589 & 1.326 & 0.841 & 2.444 & 0.644 & 1.219 & 0.507 & 3.578 & 1.581 & 4.033 & \\
\hline
\end{tabular}

Table 2. Prior information, hyper parameter values and the posterior probabilities.

\begin{tabular}{cccccccccccc}
\hline$j$ & 1 & 2 & 3 & 4 & 5 & 6 & 7 & 8 & 9 & 10 \\
\hline$\beta_{j}$ & 0.25 & 0.26 & 0.27 & 0.28 & 0.29 & 0.30 & 0.31 & 0.32 & 0.33 & 0.34 \\
$e_{j}$ & 0.1 & 0.1 & 0.1 & 0.1 & 0.1 & 0.1 & 0.1 & 0.1 & 0.1 & 0.1 \\
$a_{j}$ & 0.346 & 0.344 & 0.343 & 0.341 & 0.340 & 0.339 & 0.338 & 0.336 & 0.335 & 0.334 \\
$b_{j}$ & 0.078 & 0.075 & 0.072 & 0.069 & 0.066 & 0.064 & 0.062 & 0.059 & 0.057 & 0.056 \\
$p_{j}$ & 0.0021 & 0.0041 & 0.0078 & 0.0148 & 0.0280 & 0.0482 & 0.0822 & 0.1543 & 0.2613 & 0.3973 \\
\hline
\end{tabular}

Table 3. The ML and the Bayes estimates of $\beta, \alpha, S(t)$ and $H(t)$ with $t=0.4$.

\begin{tabular}{|c|c|c|c|c|c|c|c|c|c|}
\hline & $(.)_{M L}$ & $(.)_{B S}$ & $(.)_{B L}$ & & & & $(.)_{G E}$ & & \\
\hline & & & & $c=-1$ & $c=1$ & $c=0.0001$ & $q=-1$ & $q=1$ & $q=2$ \\
\hline$\beta$ & 0.2827 & 0.2439 & 0.3266 & 0.3267 & 0.3264 & 0.3266 & 0.3266 & 0.3257 & 0.3252 \\
\hline$\alpha$ & 0.6235 & 0.6795 & 0.8422 & 0.8667 & 0.8194 & 0.8422 & 0.8422 & 0.7863 & 0.7578 \\
\hline$S(t)$ & 0.7560 & 0.7099 & 0.7173 & 0.7191 & 0.7155 & 0.7173 & 0.7173 & 0.7120 & 0.7093 \\
\hline$H(t)$ & 1.1267 & 1.3457 & 1.3846 & 1.4512 & 1.3256 & 1.3846 & 1.3846 & 1.2943 & 1.2484 \\
\hline
\end{tabular}


tions to compare the performances of the different estimators for different sampling schemes. All the computations are performed with a Pentium IV processor using Mathematica 8.0. We used different sample sizes $(n=$ number of groups), different group sizes $(k)$, different effective sample sizes $(m)$, and different sampling schemes (i.e., different $R_{i}$ values). We used two sets of parameter values: $\beta=1, \alpha=0.5$ and $\beta=0.1, \alpha=$ 0.2 , mainly to compare the MLEs and different Bayes estimators in terms of their mean squared errors (MSEs), and also to explore their effects on different parameter values.

To generate progressive first- failure censored samples from CRD, we used the algorithm proposed by [13], with the fact that, the progressive first-failure censored sample $X_{1 ; m, n, k}^{R}, X_{2 ; m, n, k}^{R}, \cdots, X_{m ; m, n, k}^{R}$ with distribution function $F(x)$, can be viewed as a progressive type II censored sample from a population with distribution function $1-(1-F(x))^{k}$. We assume that the number of items putting in a life test is $(n \times k)$ items, where $n$ denotes the number of groups and $k$ denotes the number of items in each group. Using a progressive first-failure censoring scheme, only $m$ observations are record from the test. To compare the performances of the estimation procedures developed in this paper, we consider the following three progressive censoring schemes (C.S ), namely:

Scheme I: $R_{m}=n-m, R_{i}=0$ for $i \neq m$.
Scheme II: $R_{1}=n-m, R_{i}=0$ for $i \neq 1$.

Scheme III: $R_{\frac{m+1}{2}}=n-m, R_{i}=0$ for $i \neq \frac{m+1}{2}$; if $m$ odd, and $\frac{R_{\frac{m+2}{2}}}{2}=n-m, R_{i}=0$ for $i \neq \frac{m+2}{2}$; if
$m$ even.

The three censoring schemes are corresponding, respectively, to the cases of all surviving items are removed from the experiment at the last failure point, first failure point and midpoint. Also, it should be noted that scheme I is the Type-II first-failure censored scheme.

In Tables 4-7, we report the mean squared errors (MSEs) of the ML estimates and different Bayes estimates of the parameters, reliability and hazard rate functions, based on 1000 replications. The results are reported in Tables 4 and 5 for the parameters values $(\beta=1, \alpha=0.5)$. Tables 6 and 7 display the same results for the parameters values $(\beta=0.1, \alpha=0.2)$.

\section{Conclusions}

In this paper, the maximum likelihood and Bayes methods are used for estimating parameters, reliability function and hazard rate function of the CRD based on a new censoring scheme, called a progressive first-failure censoring scheme. Combining the concept of first-failure censoring and the concept of progressive censoring, a

Table 4. MSEs of estimates of $\beta$ and $\alpha$ when $(\beta=1, \alpha=0.5 ; c=q=1)$.

\begin{tabular}{|c|c|c|c|c|c|c|c|c|c|}
\hline$m, n, k$ & C.S & $\hat{\beta}_{M L}$ & $\hat{\beta}_{B S}$ & $\hat{\beta}_{B L}$ & $\hat{\beta}_{G E}$ & $\hat{\alpha}_{M L}$ & $\hat{\alpha}_{B S}$ & $\hat{\alpha}_{B L}$ & $\hat{\alpha}_{G E}$ \\
\hline \multirow[t]{3}{*}{$15,30,1$} & I & 4.6722 & 0.1581 & 0.1121 & 0.1580 & 0.4997 & 0.2319 & 0.2024 & 0.1801 \\
\hline & II & 3.0944 & 0.1561 & 0.1109 & 0.1558 & 0.1440 & 0.1744 & 0.1522 & 0.1334 \\
\hline & III & 3.2318 & 0.1574 & 0.1117 & 0.1572 & 0.3022 & 0.2199 & 0.1919 & 0.1702 \\
\hline \multirow[t]{3}{*}{$25,30,1$} & I & 2.0300 & 0.1596 & 0.1130 & 0.1596 & 0.1032 & 0.1341 & 0.1236 & 0.1123 \\
\hline & II & 1.6751 & 0.1595 & 0.1129 & 0.1594 & 0.0812 & 0.1323 & 0.1219 & 0.1107 \\
\hline & III & 1.7901 & 0.1596 & 0.1129 & 0.1595 & 0.0819 & 0.1406 & 0.1296 & 0.1181 \\
\hline \multirow[t]{2}{*}{$30,50,1$} & I & 3.4690 & 0.1599 & 0.1132 & 0.1599 & 0.2759 & 0.1528 & 0.1429 & 0.1323 \\
\hline & III & 1.1757 & 0.1599 & 0.1132 & 0.1599 & 0.0851 & 0.1361 & 0.1272 & 0.1173 \\
\hline \multirow[t]{3}{*}{$15,30,3$} & I & 3.3908 & 0.1591 & 0.1127 & 0.1591 & 0.6708 & 0.2536 & 0.2218 & 0.1971 \\
\hline & II & 3.0030 & 0.1583 & 0.1122 & 0.1582 & 0.3806 & 0.2389 & 0.2081 & 0.1860 \\
\hline & III & 3.4677 & 0.1587 & 0.1125 & 0.1586 & 0.5406 & 0.2651 & 0.2311 & 0.2070 \\
\hline \multirow[t]{3}{*}{$25,30,3$} & I & 3.0341 & 0.1599 & 0.1132 & 0.1599 & 0.4048 & 0.1981 & 0.1827 & 0.1684 \\
\hline & II & 3.6552 & 0.1599 & 0.1131 & 0.1598 & 0.4000 & 0.1757 & 0.1621 & 0.1487 \\
\hline & III & 3.4242 & 0.1599 & 0.1131 & 0.1599 & 0.4017 & 0.1978 & 0.1824 & 0.1685 \\
\hline \multirow[t]{2}{*}{$30,50,3$} & I & 3.1022 & 0.1600 & 0.1132 & 0.1600 & 0.4959 & 0.2220 & 0.2076 & 0.1945 \\
\hline & III & 2.8783 & 0.1600 & 0.1132 & 0.1600 & 0.4631 & 0.1883 & 0.1760 & 0.1641 \\
\hline \multirow[t]{3}{*}{$15,30,5$} & I & 2.8347 & 0.1593 & 0.1128 & 0.1592 & 0.6708 & 0.2888 & 0.2524 & 0.2262 \\
\hline & II & 3.4444 & 0.1588 & 0.1125 & 0.1587 & 0.5717 & 0.2669 & 0.2328 & 0.2086 \\
\hline & III & 3.4996 & 0.1590 & 0.1126 & 0.1590 & 0.5610 & 0.2596 & 0.2270 & 0.2024 \\
\hline \multirow[t]{3}{*}{$25,30,5$} & I & 2.6896 & 0.1599 & 0.1132 & 0.1599 & 0.4647 & 0.2185 & 0.2015 & 0.1866 \\
\hline & II & 3.4326 & 0.1599 & 0.1132 & 0.1599 & 0.5458 & 0.2093 & 0.1931 & 0.1783 \\
\hline & III & 3.1225 & 0.1599 & 0.1132 & 0.1599 & 0.4743 & 0.2015 & 0.1859 & 0.1714 \\
\hline \multirow[t]{3}{*}{$30,50,5$} & I & 3.0823 & 0.1600 & 0.1132 & 0.1600 & 0.5514 & 0.2084 & 0.1950 & 0.1820 \\
\hline & II & 3.0104 & 0.1600 & 0.1132 & 0.1600 & 0.4230 & 0.1884 & 0.1762 & 0.1641 \\
\hline & III & 2.7733 & 0.1600 & 0.1132 & 0.1600 & 0.5472 & 0.204 & 0.1908 & 0.1782 \\
\hline
\end{tabular}


Table 5. MSEs of estimates of $S(t)$ and $H(t)$ when $(\beta=1, \alpha=0.5 ; c=q=1$ and $t=1.5)$.

\begin{tabular}{|c|c|c|c|c|c|c|c|c|c|}
\hline$m, n, k$ & C.S & $\hat{S}_{M L}$ & $\hat{S}_{B S}$ & $\hat{S}_{B L}$ & $\hat{S}_{G E}$ & $\hat{H}_{M L}$ & $\hat{H}_{B S}$ & $\hat{H}_{B L}$ & $\hat{H}_{G E}$ \\
\hline \multirow[t]{3}{*}{$15,30,1$} & I & 0.0082 & 0.0217 & 0.0226 & 0.0275 & 0.0414 & 0.1260 & 0.1113 & 0.0958 \\
\hline & II & 0.0118 & 0.0165 & 0.0172 & 0.0211 & 0.0220 & 0.0928 & 0.0819 & 0.0696 \\
\hline & III & 0.0085 & 0.0206 & 0.0215 & 0.0262 & 0.0343 & 0.1190 & 0.1051 & 0.0902 \\
\hline \multirow[t]{3}{*}{$25,30,1$} & I & 0.0062 & 0.0129 & 0.0134 & 0.0153 & 0.0123 & 0.0665 & 0.0616 & 0.0544 \\
\hline & II & 0.0074 & 0.0127 & 0.0131 & 0.0151 & 0.0113 & 0.0656 & 0.0607 & 0.0537 \\
\hline & III & 0.0067 & 0.0137 & 0.0141 & 0.0162 & 0.0127 & 0.0706 & 0.0654 & 0.0581 \\
\hline \multirow[t]{2}{*}{$30,50,1$} & I & 0.0043 & 0.0152 & 0.0156 & 0.0175 & 0.0202 & 0.0767 & 0.0719 & 0.0651 \\
\hline & III & 0.0046 & 0.0133 & 0.0137 & 0.0154 & 0.0114 & 0.0672 & 0.063 & 0.0567 \\
\hline \multirow[t]{3}{*}{$15,30,3$} & I & 0.0087 & 0.0239 & 0.0250 & 0.0305 & 0.0598 & 0.1375 & 0.1216 & 0.1044 \\
\hline & II & 0.0076 & 0.0218 & 0.0227 & 0.0277 & 0.0406 & 0.1303 & 0.1148 & 0.0994 \\
\hline & III & 0.0077 & 0.0244 & 0.0254 & 0.031 & 0.0514 & 0.1453 & 0.1281 & 0.1111 \\
\hline \multirow[t]{3}{*}{$25,30,3$} & I & 0.0048 & 0.0197 & 0.0203 & 0.0231 & 0.0364 & 0.1030 & 0.0955 & 0.0858 \\
\hline & II & 0.0046 & 0.0174 & 0.018 & 0.0205 & 0.0289 & 0.0901 & 0.0835 & 0.0747 \\
\hline & III & 0.0053 & 0.0195 & 0.02 & 0.0228 & 0.0336 & 0.1035 & 0.096 & 0.0866 \\
\hline \multirow[t]{2}{*}{$30,50,3$} & I & 0.0056 & 0.0227 & 0.0233 & 0.0259 & 0.044 & 0.1166 & 0.1095 & 0.1005 \\
\hline & III & 0.0048 & 0.0189 & 0.0194 & 0.0217 & 0.0372 & 0.0970 & 0.0911 & 0.0831 \\
\hline \multirow[t]{3}{*}{$15,30,5$} & I & 0.0113 & 0.0272 & 0.0283 & 0.0344 & 0.0687 & 0.1587 & 0.1403 & 0.1216 \\
\hline & II & 0.0083 & 0.0245 & 0.0256 & 0.0312 & 0.0586 & 0.1464 & 0.1291 & 0.112 \\
\hline & III & 0.0086 & 0.0245 & 0.0255 & 0.0311 & 0.0563 & 0.1416 & 0.1252 & 0.1079 \\
\hline \multirow[t]{3}{*}{$25,30,5$} & I & 0.0063 & 0.0217 & 0.0223 & 0.0254 & 0.0468 & 0.1153 & 0.1069 & 0.0967 \\
\hline & II & 0.0056 & 0.0209 & 0.0215 & 0.0245 & 0.0464 & 0.1096 & 0.1017 & 0.0916 \\
\hline & III & 0.0052 & 0.0202 & 0.0208 & 0.0236 & 0.0401 & 0.1050 & 0.0974 & 0.0875 \\
\hline \multirow[t]{3}{*}{$30,50,5$} & I & 0.0066 & 0.0214 & 0.0220 & 0.0245 & 0.0504 & 0.1082 & 0.1016 & 0.0928 \\
\hline & II & 0.0046 & 0.0191 & 0.0196 & 0.0219 & 0.0368 & 0.0968 & 0.0909 & 0.0828 \\
\hline & III & 0.0058 & 0.0208 & 0.0214 & 0.0238 & 0.0480 & 0.1060 & 0.0996 & 0.0911 \\
\hline
\end{tabular}

Table 6. MSEs of estimates $\beta$ and $\alpha$ with $(\beta=0.1, \alpha=0.2 ; c=q=1)$.

\begin{tabular}{|c|c|c|c|c|c|c|c|c|c|}
\hline$m, n, k$ & C.S & $\hat{\beta}_{M L}$ & $\hat{\beta}_{B S}$ & $\hat{\beta}_{B L}$ & $\hat{\beta}_{G E}$ & $\hat{\alpha}_{M L}$ & $\hat{\alpha}_{B S}$ & $\hat{\alpha}_{B L}$ & $\hat{\alpha}_{G E}$ \\
\hline \multirow[t]{3}{*}{$15,30,1$} & I & 0.64310 & 0.02612 & 0.02609 & 0.02579 & 0.44127 & 0.07859 & 0.07895 & 0.08516 \\
\hline & II & 0.22796 & 0.02602 & 0.02599 & 0.02564 & 0.11098 & 0.08074 & 0.08111 & 0.08737 \\
\hline & III & 0.38175 & 0.02631 & 0.02628 & 0.02592 & 0.24153 & 0.08009 & 0.08041 & 0.08641 \\
\hline \multirow[t]{3}{*}{$25,30,1$} & I & 0.18708 & 0.02508 & 0.02507 & 0.02501 & 0.06972 & 0.08116 & 0.08141 & 0.08544 \\
\hline & II & 0.19074 & 0.02507 & 0.02506 & 0.02499 & 0.06741 & 0.08038 & 0.08063 & 0.08468 \\
\hline & III & 0.16512 & 0.02508 & 0.02508 & 0.02501 & 0.07145 & 0.08094 & 0.08118 & 0.08517 \\
\hline \multirow[t]{2}{*}{$30,50,1$} & I & 0.15809 & 0.02502 & 0.02502 & 0.02500 & 0.08661 & 0.08094 & 0.08070 & 0.08407 \\
\hline & III & 0.11434 & 0.02504 & 0.02504 & 0.02500 & 0.07154 & 0.08050 & 0.08071 & 0.08413 \\
\hline \multirow[t]{3}{*}{$15,30,3$} & I & 0.68345 & 0.02777 & 0.02773 & 0.02720 & 0.77036 & 0.07411 & 0.07444 & 0.08043 \\
\hline & II & 0.62398 & 0.02650 & 0.02647 & 0.02611 & 0.52082 & 0.07623 & 0.07660 & 0.08283 \\
\hline & III & 0.66446 & 0.02716 & 0.02712 & 0.02677 & 0.84312 & 0.07416 & 0.07448 & 0.08034 \\
\hline \multirow[t]{3}{*}{$25,30,3$} & I & 0.43638 & 0.02530 & 0.02529 & 0.02522 & 0.39264 & 0.07376 & 0.07406 & 0.07812 \\
\hline & II & 0.24410 & 0.02524 & 0.02523 & 0.02516 & 0.20388 & 0.07612 & 0.07637 & 0.08041 \\
\hline & III & 0.25466 & 0.02530 & 0.02529 & 0.02521 & 0.25822 & 0.07756 & 0.07781 & 0.08179 \\
\hline \multirow[t]{2}{*}{$30,50,3$} & I & 0.36434 & 0.02527 & 0.02526 & 0.02521 & 0.4516 & 0.07262 & 0.07285 & 0.0764 \\
\hline & III & 0.16665 & 0.02519 & 0.02519 & 0.02514 & 0.18583 & 0.07255 & 0.07278 & 0.07627 \\
\hline \multirow[t]{3}{*}{$15,30,5$} & I & 0.61095 & 0.02777 & 0.02772 & 0.02719 & 0.76829 & 0.07481 & 0.07512 & 0.08099 \\
\hline & II & 0.6123 & 0.02701 & 0.02697 & 0.02655 & 0.60319 & 0.07517 & 0.07552 & 0.08171 \\
\hline & III & 0.54737 & 0.02773 & 0.02768 & 0.02716 & 0.71943 & 0.07238 & 0.07271 & 0.07876 \\
\hline \multirow[t]{3}{*}{$25,30,5$} & I & 0.44526 & 0.0256 & 0.02559 & 0.02547 & 0.49019 & 0.07199 & 0.07225 & 0.07636 \\
\hline & II & 0.35068 & 0.02542 & 0.02541 & 0.02532 & 0.37164 & 0.07434 & 0.0746 & 0.07868 \\
\hline & III & 0.36293 & 0.02552 & 0.02551 & 0.0254 & 0.39279 & 0.07088 & 0.07115 & 0.07531 \\
\hline \multirow[t]{3}{*}{$30,50,5$} & I & 0.68456 & 0.02557 & 0.02557 & 0.02546 & 0.88917 & 0.06958 & 0.06982 & 0.07339 \\
\hline & II & 0.37034 & 0.02519 & 0.02519 & 0.02515 & 0.40385 & 0.07401 & 0.07424 & 0.07772 \\
\hline & III & 0.38334 & 0.02534 & 0.02533 & 0.02527 & 0.57943 & 0.07144 & 0.07168 & 0.07168 \\
\hline
\end{tabular}

progressive first-failure censoring scheme has been introduced by [21]. This censoring scheme has advantages in terms of reducing test time, in which more items are used but only $m$ of $(n \times k)$ items are failures. Based on this new censoring scheme, the present paper shows how the things can be routinely managed for the CRD in a 
Table 7. MSEs of estimates of $S(t)$ and $H(t)$ when $(\beta=0.1, \alpha=0.2 ; c=q=1$ and $t=1.5)$.

\begin{tabular}{|c|c|c|c|c|c|c|c|c|c|}
\hline$m, n, k$ & C.S & $\hat{S}_{M L}$ & $\hat{S}_{B S}$ & $\hat{S}_{B L}$ & $\hat{S}_{G E}$ & $\hat{H}_{M L}$ & $\hat{H}_{B S}$ & $\hat{H}_{B L}$ & $\hat{H}_{G E}$ \\
\hline \multirow{3}{*}{$15,30,1$} & I & 0.104547 & 0.17122 & 0.16955 & 0.16638 & 0.24275 & 0.10171 & 0.1023 & 0.1101 \\
\hline & II & 0.0966954 & 0.17595 & 0.17429 & 0.17113 & 0.10649 & 0.10446 & 0.10506 & 0.11292 \\
\hline & III & 0.0996995 & 0.17337 & 0.17174 & 0.16872 & 0.1746 & 0.10357 & 0.1041 & 0.11165 \\
\hline \multirow[t]{3}{*}{$25,30,1$} & I & 0.07744 & 0.1756 & 0.17453 & 0.1725 & 0.07316 & 0.10507 & 0.10547 & 0.11049 \\
\hline & II & 0.08063 & 0.17389 & 0.17281 & 0.17076 & 0.06942 & 0.10407 & 0.10448 & 0.10953 \\
\hline & III & 0.07749 & 0.17502 & 0.17396 & 0.17195 & 0.07716 & 0.10407 & 0.10516 & 0.11015 \\
\hline \multirow[t]{3}{*}{$30,50,1$} & I & 0.06926 & 0.17383 & 0.17293 & 0.17123 & 0.08715 & 0.10422 & 0.10455 & 0.10877 \\
\hline & II & 0.07092 & 0.17664 & 0.17573 & 0.17403 & 0.06067 & 0.10601 & 0.10634 & 0.11059 \\
\hline & III & 0.06447 & 0.17376 & 0.17285 & 0.17112 & 0.07603 & 0.10425 & 0.10459 & 0.10886 \\
\hline \multirow[t]{3}{*}{$15,30,3$} & I & 0.16069 & 0.16155 & 0.15989 & 0.15675 & 0.48559 & 0.096 & 0.09654 & 0.1041 \\
\hline & II & 0.11215 & 0.16632 & 0.16464 & 0.1614 & 0.27853 & 0.09873 & 0.09932 & 0.10716 \\
\hline & III & 0.1446 & 0.16122 & 0.15959 & 0.15652 & 0.47758 & 0.096 & 0.09653 & 0.10394 \\
\hline \multirow[t]{3}{*}{$25,30,3$} & I & 0.10164 & 0.16041 & 0.15929 & 0.15712 & 0.23557 & 0.09566 & 0.09609 & 0.1012 \\
\hline & II & 0.08286 & 0.16497 & 0.16387 & 0.16176 & 0.15397 & 0.09864 & 0.09906 & 0.10411 \\
\hline & III & 0.09343 & 0.16806 & 0.16699 & 0.16493 & 0.19534 & 0.10047 & 0.10087 & 0.10585 \\
\hline \multirow[t]{3}{*}{$30,50,3$} & I & 0.12342 & 0.1577 & 0.15673 & 0.15483 & 0.32138 & 0.09427 & 0.09496 & 0.09906 \\
\hline & II & 0.07157 & 0.1655 & 0.16456 & 0.16275 & 0.11354 & 0.09909 & 0.09945 & 0.10379 \\
\hline & III & 0.09084 & 0.15749 & 0.15653 & 0.15465 & 0.17242 & 0.09415 & 0.09452 & 0.09888 \\
\hline \multirow{3}{*}{$15,30,5$} & I & 0.015722 & 0.16256 & 0.16092 & 0.15786 & 0.47972 & 0.09686 & 0.09737 & 0.1048 \\
\hline & II & 0.01313 & 0.16406 & 0.16237 & 0.15913 & 0.36439 & 0.09738 & 0.09796 & 0.10574 \\
\hline & III & 0.015951 & 0.15781 & 0.15612 & 0.15291 & 0.47296 & 0.0938 & 0.09436 & 0.10198 \\
\hline \multirow{3}{*}{$25,30,5$} & I & 0.013146 & 0.15666 & 0.15553 & 0.15332 & 0.3405 & 0.09342 & 0.09385 & 0.09899 \\
\hline & II & 0.10276 & 0.16138 & 0.16026 & 0.1581 & 0.23814 & 0.0964 & 0.09682 & 0.10193 \\
\hline & III & 0.11713 & 0.15424 & 0.15309 & 0.15082 & 0.27399 & 0.09202 & 0.09246 & 0.09766 \\
\hline \multirow{3}{*}{$30,50,5$} & I & 0.1593 & 0.15165 & 0.15066 & 0.14871 & 0.49866 & 0.09042 & 0.09081 & 0.09526 \\
\hline & II & 0.10173 & 0.16047 & 0.15951 & 0.15766 & 0.24817 & 0.09601 & 0.09638 & 0.10073 \\
\hline & III & 0.11933 & 0.15529 & 0.15432 & 0.15241 & 0.32279 & 0.09276 & 0.09314 & 0.9754 \\
\hline
\end{tabular}

Bayesian and classical frameworks. We have considered the maximum likelihood (ML) and Bayes estimates for the parameters of the CRD, as well as some survival time parameters, reliability and hazard rate functions using progressively first-failure censored data. The Bayes estimators are discussed under symmetric and asymmetric loss functions. The use of a discrete prior distribution for parameter $\beta$ resulted in a closed form expression for the posterior pdf, and the equal probabilities in the discrete distribution cased an element of uncertainly, which can be desirable in some cases. All of the results obtained in this article can be specialized to: a): the firstfailure censored data when $\mathbf{R}=\{0,0, \cdots, 0\} . b)$ : the progressive type II censored order statistics if $k=1$. c): Type II censored order statistics when $k=1$ and $\mathbf{R}=\{0,0, \cdots, n-m\}$.d): the complete sample case when $k=1$ and $\mathbf{R}=\{0,0, \cdots, 0\}$. A simulation study was conducted to examine the performance of the different estimators. From the results, we observe the following:

- The Bayes estimates are better than the MLEs in general, and the Bayes estimates relative to asymmetric loss functions (LINEX and GE loss) performed better than the others in the sense of comparing the MSE of the estimates. This was true for all censored schemes.

- From all Tables, as the effective sample proportion $\mathrm{m} / \mathrm{n}$ increases, the MSE of the estimators, reduce significantly. Concerning a progressive type-II cen- soring scheme $(k=1)$, for a fixed $m$ and $n$, we can determine the censoring scheme $(\mathbf{R})$, which is most efficient; for example, in all tables it is seen that the case of scheme II (all items removed at the first failure time point), with $(k=1)$ is better than the other cases in the sense of comparing the MSEs.

- To access the effect of the shape parameters of the asymmetric loss functions $c$ and $q$, we examine different values of $c$ and $q$ we see that if $c$ is near to 0 , and $q=-1$, then the Bayes estimates are almost the same as the estimates under SEL, see Table 3. This is one of the useful properties of working with the asymmetric loss functions.

- The results establish that for optimum decision making, important should be given on the choice of loss function and not just the choice of prior distribution only.

- The simulation study shows that the MSEs for all estimates are increases as the value of the shape and scale parameters increases.

\section{References}

[1] R. Viveros and N. Balakrishnan, "Interval Estimation of Pa- rameters of Life from Progressively Censored Data," Te- chnometrics, Vol. 36, No. 1, 1994, pp. 84-91. doi:10.2307/1269201

[2] N. Balakrishnan and R. A. Sandhu, "A Simple Simulation 
Algorithm for Generating Progressively Type-II Censored Samples," The American Statistician, Vol. 49, No. 2, 1995, pp. 229-230. doi:10.2307/2684646

[3] N. Balakrishnan and R. A. Sandhu, "Best Linear Unbiased and Maximum Likelihood Estimation for Exponential Distributions under General Progressive Type-II Censored Samples," Sankhya: The Indian Journal of Seriestics, Vol. 58, No. 1, 1996, pp. 1-9.

[4] P. Mostert, J. Roux and A. Bekker, "Bayes Estimators of the Life Time Parameters Using the Compound Rayleigh model," Journal of South African Statistical Association, Vol. 33, No. 2 ,1999, pp. 117-138.

[5] U. Balasooriya and N. Balakrishnan, "Reliability Sampling Plans for Log-Normal Distribution Based on Progressively Censored Samples," IEEE Transactions on Reliability, Vol. 49, No. 2, 2000, pp. 199-203. doi:10.1109/24.877338

[6] A. Bekker, J. Roux and P. Mostert, "A Generalization of the Compound Rayleigh Distribution: Using a Bayesian Methods on Cancer Survival Times," Communications in Statistics-Theory and Methods, Vol. 29, No. 7, 2000, pp. 1419-1433. doi:10.1080/03610920008832554

[7] H. K. T. Ng, P. S. Chan and N. Balakrishnan, "Optimal Progressive Censoring Plans for the Weibull Distribution," Technometrics, Vol. 46, No. 4, 2004, pp. 470-481. doi:10.1198/004017004000000482

[8] H. K. T. Ng, "Parameter estimation for a modifiied Weibull Distribution, for Progressively Type-II Censored Samples," IEEE Transactions Reliability, Vol. 54, No. 3, 2005, pp. 374-380. doi:10.1109/TR.2005.853036

[9] N. Balakrishnan, N. Kannan, C. T. Lin and H. K. T. Ng, "Point and Interval Estimation for Gaussian Distribution Based on Progressively Type-II Censored Samples," IEEE Transactions on Reliability, Vol. 52, No. 1, 2003, pp. 90-95. doi:10.1109/TR.2002.805786

[10] A. A. Soliman, "Estimation of Parameters of Life from Progressively Censored Data Using Burr-XII Model," IEEE Transactions Reliability, Vol. 54, No. 1, 2005, pp. 34-42. doi:10.1109/TR.2004.842528

[11] A. A. Soliman, "Estimation for Pareto Model Using General Progressive Censored Data and Asymmetric Loss," Communications in Statistics - Theory and Methods, Vol. 37, No. 9, 2008, pp. 1353-1370. doi:10.1080/03610920701825957

[12] D. G. Chen and Y. L. Lio, "Parameter Estimations for Generalized Exponential Distribution under Progressive Type-I Interval Censoring," Computational Statistics and Data Analysis, Vol. 54, No. 6, 2010, pp. 1581-1591. doi:10.1016/j.csda.2010.01.007

[13] N. Balakrishnan and R. Aggarwala, "Progressive Censoring-Theory, Methods and Applications," Birkhäuser, Boston, 2000.

[14] N. Balakrishnan, "Progressive Censoring Methodology: An Appraisal," Test, Vol. 16, No. 2007, pp. 211-289. doi:10.1007/s11749-007-0061-y

[15] L. G. Johnson, "Theory and Technique of Variation Research," Elsevier, Amsterdam, 1964.
[16] C.-H. Jun, S. Balamurali and S.-H. Lee, "Variables Sampling Plans for Weibull Distributed Lifetimes under Sudden Death Testing," IEEE Transactions on Reliability, Vol. 55, No. 1, 2006, pp. 53-58. doi:10.1109/TR.2005.863802

[17] J.-W. Wu, W.-L. Hung and C.-H. Tsai, "Estimation of the Parameters of the Gompertz Distribution under the First Failure-Censored Sampling Plan," Statistics, Vol. 37, No. 6, 2003, pp. 517-525. doi: $10.1080 / 02331880310001598864$

[18] J.-W. Wu and H. -Y. Yu, "Statistical Inference about the Shape Parameter of the Burr Type XII Distribution under the Failure-Censored Sampling Plan," Applied Mathematics and computation, Vol. 163, No. 1, 2005, pp. 443482. doi:10.1016/j.amc.2004.02.019

[19] J.-W. Wu, T.-R. Tsai and L.-Y. Ouyang, "Limited Failure-Censored Life Test for the Weibull Distribution," IEEE Transactions on Reliability, Vol. 50, No. 1, 2001, pp. 107-111. doi:10.1109/24.935024

[20] W.-C. Lee, L.-W. Wu and H.-Y. Yu, "Statistical Inference about the Shape Parameter of the Bathtub-Shaped Distribution under the Failure-Censored Sampling Plan," International Journal of Information and Management Sciences, Vol. 18, No. 2, 2007, pp. 157-172.

[21] S.-J. Wu and C. Kus, "On Estimation Based on Progressive First Failure-Censored Sampling," Computational Statistics and Data Analysis, Vol. 53, No. 10, 2009, pp. $1-12$.

[22] E. K. AL-Hussaini and Z. F. Jaheen, "Bayes Estimation of the Parameters, Reliability and Failure Rate Functions of the Burr Type XII Failure Model," Journal of Statistics Computation and Simulation, Vol. 44, 1992, pp. 31-40.

[23] E. K. AL-Hussaini and Z. F. Jaheen, “Approximate Bayes Estimators Applied to the Burr Model," Communications in Statistics, Vol. 23, 1994, pp. 99-121.

[24] E. K. AL-Hussaini and Z. F. Jaheen, "Bayesian Prediction Bounds for the Burr XII Model," Communications in Statistics, Vol. 24, 1995, pp. 1829-1842.

[25] E. K. AL-Hussaini and Z. F. Jaheen, "Bayesian Prediction Bounds for the Burr Type XII Distribution in the Presence of Outliers," Journal of Statistical Planning and Inference, Vol. 55, No. 1, 1996, pp. 23-37. doi:10.1016/0378-3758(95)00184-0

[26] E. K. AL-Hussaini, "Predicting Observables from a General Class of Distributions," Journal of Statistical Planning and Inference, Vol. 79, No. 1, 1999, pp. 79-91. doi:10.1016/S0378-3758(98)00228-6

[27] N. Balakrishnan and M. Kateri, "Statistical Evidence in Contingency Tables Analysis," Journal of Statistical Planning and Inference, Vol. 138, No. 4, 2008, pp. 873- 887. doi:10.1016/j.jspi.2007.02.005

[28] H. R. Varian, "A Bayesian Approach to Real State Assessment," In: E. F. Stephen and A. Zellner, Eds., Studies in Bayesian Econometrics and Statistics in Honor of Leonard J. Savage, North-Holland, Amsterdam, 1975, pp. 195-208.

[29] X. Y. Li, Y. Shi, J. Wei and J. Chai, "Empirical Bayes 
Estimators of Reliability Performances Using LINEX loss under Progressively Type-II Censored Samples," Mathematics and Computers in Simulation, Vol. 73, No. 5, 2007, pp. 320-326. doi:10.1016/j.matcom.2006.05.002

[30] G. Prakash and D. C. Singh, "Shrinkage Estimation in Exponential Type-II Censored Data under LINEX Loss," Journal of the Korean Statistical Society, Vol. 37, No. 1, 2008, pp. 53-61. doi:10.1016/j.jkss.2007.07.002

[31] D. K. Day, M. Ghosh and C. Srinivasan, "Simultaneous Estimation of Parameters under Entropy Loss," Journal of Statistical Planning and Inference, Vol. 52, 1987, pp. 347-363. doi:10.1016/0378-3758(86)90108-4

[32] D. K. Day and P. L. Liu, "On Comparison of Estimation in Generalized Life Model," Microelectronics Reliability, Vol. 32, No. 1-2, 1992, pp. 207-221. doi:10.1016/0026-2714(92)90099-7
[33] R. M. Soland, "Bayesian analysis of the Weibull Process with Unknown Scale and Shape Parameters," IEEE Transactions on Reliability, Vol. 18, No. 4, 1969, pp. 181-184. doi:10.1109/TR.1969.5216348

[34] A. A. Soliman, A. H. Abd Ellah and K. S. Sultan, "Comparison of Estimates Using Record Statistics from Weibull Model: Bayesian and Non-Bayesian Approaches," Computational Statistics \& Data Analysis, Vol. 51, No. 3, 2006, pp. 2065-2077. doi:10.1016/j.csda.2005.12.020

[35] H. F. Martz and R. A. Waller, "Bayesian Reliability Analysis," Wiley, New York, 1982.

[36] D. M. Stablein, W. H. Carter and J. W. Novak, "Analysis of Survival Data with Non-Proportional Hazard Functions," Controlled Clinical Trials, Vol. 2, No. 2, 1981, pp. 149-159. doi:10.1016/0197-2456(81)90005-2 